\title{
A Printable and Conductive Yield-Stress Fluid as an Ultrastretchable Transparent Conductor
}

\author{
Qianying Lu $\mathbb{D}^{1},{ }^{1}$ Yunlei Zhou, ${ }^{1}$ Xiangfei Yin $\mathbb{D}^{2},{ }^{2}$ Shitai Cao, ${ }^{1}$ Xiaoliang Wang $\mathbb{D},{ }^{2}$ \\ and Desheng Kong $\mathbb{D}^{1}$ \\ ${ }^{1}$ College of Engineering and Applied Sciences, State Key Laboratory of Analytical Chemistry for Life Science, and Jiangsu Key \\ Laboratory of Artificial Functional Materials, Nanjing University, Nanjing 210046, China \\ ${ }^{2}$ Key Laboratory of High Performance Polymer Materials and Technology of Ministry of Education, Department of Polymer Science \\ and Engineering, School of Chemistry and Chemical Engineering, Nanjing University, Nanjing 210046, China
}

Correspondence should be addressed to Xiaoliang Wang; wangxiaoliang@nju.edu.cn

and Desheng Kong; mg1834043@smail.nju.edu.cn

Received 14 August 2021; Accepted 14 November 2021; Published 14 December 2021

Copyright (c) 2021 Qianying Lu et al. Exclusive Licensee Science and Technology Review Publishing House. Distributed under a Creative Commons Attribution License (CC BY 4.0).

\begin{abstract}
In contrast to ionically conductive liquids and gels, a new type of yield-stress fluid featuring reversible transitions between solid and liquid states is introduced in this study as a printable, ultrastretchable, and transparent conductor. The fluid is formulated by dispersing silica nanoparticles into the concentrated aqueous electrolyte. The as-printed features show solid-state appearances to allow facile encapsulation with elastomers. The transition into liquid-like behavior upon tensile deformations is the enabler for ultrahigh stretchability up to the fracture strain of the elastomer. Successful integrations of yield-stress fluid electrodes in highly stretchable strain sensors and light-emitting devices illustrate the practical suitability. The yield-stress fluid represents an attractive building block for stretchable electronic devices and systems in terms of giant deformability, high ionic conductivity, excellent optical transmittance, and compatibility with various elastomers.
\end{abstract}

\section{Introduction}

The rapid proliferation and evolution of consumer electronics have stimulated the growth of stretchable electronic technology featuring compliant mechanical properties [1-3]. Stretchable devices are able to be bent, twisted, stretched, and interfaced with moving objects, which are readily integrated into various emerging fields including health monitoring systems [4-6], advanced prosthetics [7-9], and human-machine interfaces $[10,11]$. Among various building materials, the stretchable transparent conductor is a key enabler for deformable forms of optoelectronic devices such as light-emitting devices [12-16] and photodetectors [17]. The prototypical forms of stretchable transparent electrodes are electronic conductors of sliver nanowires randomly assembled over elastomer substrates to form percolation conductive networks $[12,18]$. The degradations of optoelectronic performances upon tensile deformations are ascribed to the sliding between silver nanowires and damages to internanowire junctions $[19,20]$. Sliver nanowires with high area loading and plenty of junctions are often employed to achieve improved stretchability at the price of reduced optical transmittance [19-21]. In addition, the conductive properties of these electrodes typically show rapid deteriorations under biaxial tensions unless carefully engineered microstructures are employed [22, 23].

Although transparent electronic conductors struggle to achieve large deformability, the ionic counterparts have already demonstrated the suitability for the application settings of stretchable electronics. Liquid-state ionic conductors, such as ionic liquids and electrolyte solutions, embedded in elastomers represent a class of ionically conductive materials with the ultimate compliance and stretchability [24-26], The device fabrication process typically requires microfabricated molds to confine the readily flowable liquids [27]. Ionically conductive gels, formed by immobilizing liquid state conductors inside crosslinked polymer matrices, show improved processability as solid-state materials, which are compatible with various patterning techniques including photocrosslinking $[28,29]$, laser cutting $[30,31]$, and screen printing 
[32]. The potential fracture of the polymer matrix upon tensile deformations, however, requires substantial efforts to design the network structure for large stretchability [33, 34]. In addition, special surface modifications on elastomer substrates are often necessary to establish a stable and strong interface with these gels for the fabrication of functional devices, in order to prevent interfacial delamination at large tensile strains $[35,36]$.

A class of non-Newtonian fluids known as yield-stress fluids represents attractive soft materials seamlessly combining the attributes of solids and liquids [37], which are the enabler for a broad range of applications including food products [38, 39], three-dimensional printings [40-42], and drug delivery systems $[43,44]$. These materials maintain the shape with solid-state appearances at static conditions and readily flow when the applied stress exceeds a critical value. A rich variety of chemistries and structural designs are available to formulate functional, including colloid suspensions, emulsions, entangled polymer liquids, and electro/magnetorheological fluids $[45,46]$. The fully reversible solid-to-liquid transitions of yield-stress fluids allow large deformations by flowing in liquid states and thorough recovery of solid properties irrespective of the strain history [37, 45]. These desirable characteristics have rarely been harnessed in stretchable electronic devices.

In this study, we introduce a highly conductive yieldstress fluid as the printable ink for stretchable transparent conductors. The rheological complex fluid consists of colloid silica nanoparticle suspension in highly concentrated aqueous electrolyte, which is compatible with the screen printing technique for the deposition of delicate and arbitrary features over various elastomers. The as-printed features exhibit a solid appearance to enable subsequent encapsulation in elastomers. The transition into liquid-like behavior of yield-stress fluid upon tensile deformation results in conformal interactions with the elastomeric matrix and ultrahigh stretchability up to the fracture strain of the elastomer. The successful implementation of these transparent electrodes in wearable strain sensors and stretchable light-emitting devices demonstrates the practical suitability. The yield-stress fluids reported here features giant stretchability, high ionic conductivity, excellent transparency, exceptional durability, and facile integration with elastomeric structures, which represents an enticing building block for deformable optoelectronic devices and systems.

\section{Results}

An ionically conductive liquid is prepared by dissolving high concentration $\mathrm{LiCl}$ salt in an aqueous $\mathrm{PEO}$ polymer solution, which generates droplets by squeezing it out from a fine needle (see Figure 1(a)). A yield-stress fluid is formulated by further dispersing hydrophilic fumed silica into the conductive liquid and behaves like a solid-state gel under static conditions. In the extrusion process from a needle, the yieldstress fluid transforms into the flowable state and thereby forms a continuous filament (Figure 1(b)). The reversible transitions between liquid and solid states are further revealed by manipulating the fluid in static and shaking con- ditions, as shown in Movie S1. The solid behavior of the yield-stress fluid under stationary conditions is associated with the formation of a three-dimensional network of chain-type silica aggregates [47]. The network is easily disrupted under the shear stress and rapidly recovered when the external loading is removed [48]. Notice that the PEO effectively enhances the stability of the yield-stress fluid to suppress the potential sedimentation during long-term storage (see Figure S1). The rheological characteristics are systematically characterized by a Hakke RheoStress 600 rheometer, as shown in Figures 1(c) and 1(d). The conductive liquid is essentially a Newtonian fluid with a low viscosity $(\eta)$ of $\sim 1 \mathrm{~Pa} \cdot \mathrm{s}$ almost independent of the shear rate. The liquid characteristic is confirmed by the fact that the storage modulus $\left(G^{\prime}\right)$ is lower than the loss modulus $\left(G^{\prime \prime}\right)$ over the entire range of the applied shear stress (see Figure $1(\mathrm{~d})$ ). In contrast, the addition of fumed silica effectively increases the viscosity to $4 \times 10^{3} \mathrm{~Pa} \cdot \mathrm{s}$ at the quasi-static condition of $0.1 \mathrm{~s}^{-1}$. The viscosity shows a strong shear-thinning behavior with rapid reduction by over 2 orders of magnitude by increasing the shear rate from 0.1 to $200 \mathrm{~s}^{-1}$. The ink also exhibits a plateau value of $G^{\prime} \sim 8.9 \times 10^{3} \mathrm{~Pa}$, which is more than 15 times the value of $G^{\prime \prime}$ to suggest a solid-like behavior in stationary conditions. The moduli curves have a crossover point of the shear yield stress $\left(\tau_{y}\right)$ at $900 \mathrm{~Pa}$, corresponding to the reversible transition between solid and liquid states. The rheological properties of the fluids containing varying concentrations of fumed silica are further measured and shown in Figure S2. The additional silica increases viscosity, quasistatic storage moduli, and shear yield stress by forming dense network structures. In addition, the combined rheological modifiers of PEO and fume silica synergistically enhance the storage modulus and the shear yield stress (see Figure S3). In the yield-stress fluid, the polymer chains of PEO are physically absorbed onto silica surfaces and thereby strengthen the interparticle interactions of the threedimensional colloid network $[49,50]$.

The yield-stress fluid is well suited as the ink for screen printing that behaves like a low-viscosity liquid under high shear when pressed through the stencil openings, followed by rapid transformation back into solid structures to prevent spreading in the absence of additional shear [51-54]. As illustrated in Figures 1(e), a representative rose-shaped pattern is printed onto a TPU substrate exhibiting intact morphology and smooth edges. Notice that the fluid is colored in red with ponceau $4 \mathrm{R}$ dye to facilitate visual identifications. In Figure 1(f), an array of line-shaped patterns is created to reveal the minimum feature dimension as $\sim 200 \mu \mathrm{m}$, thereby demonstrating the suitability for printing delicate patterns. The rheological characteristics of the yield-stress fluid largely dictate the printing quality. The partially collapsed feature is observed with fluid formulations containing an insufficient amount of fumed silica (see Figure S4). The excellent replication of the stencil mask requires large viscosity and storage moduli with a high concentration of silica modifier to suppress the lateral spreading of the printed features (see Figure S4 and Figure S5). In addition 

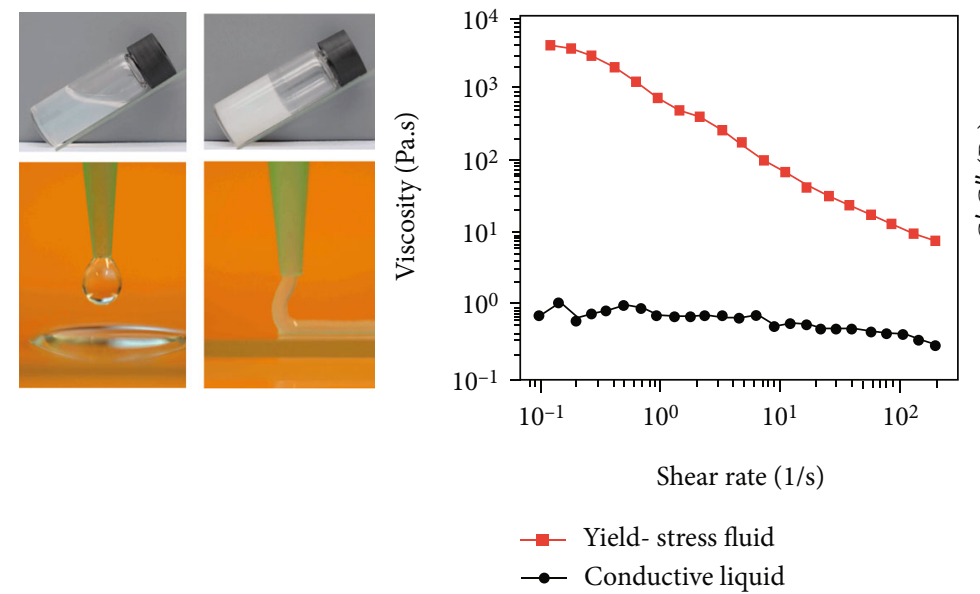

(a)

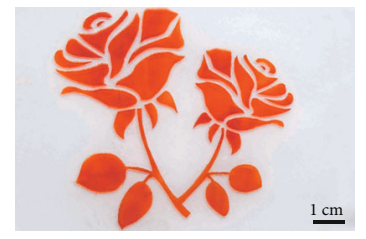

(e) (b)

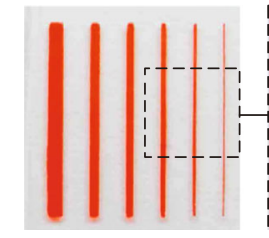

(f)



- - G' Yield- stress fluid

$\square$ G" Yield- stress fluid

- G' Conductive liquid

- - G" Conductive liquid

(c)
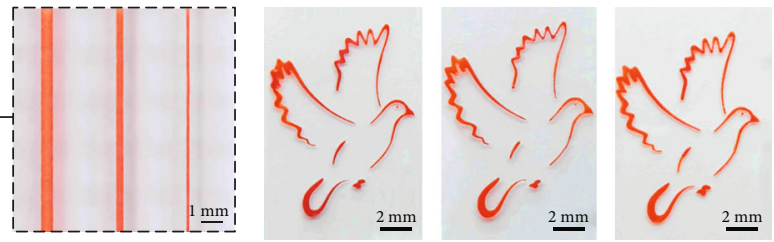

(g)

FIGURE 1: Formulation of yield-stress fluid as printable ink. (a) Ionically conductive liquid loaded in a vial (top) and squeezed through a needle (bottom). (b) Yield-stress fluid loaded in a vial (top) and extruded through a needle (bottom). (c) Viscosity versus shear rate for the conductive liquid and the yield-stress fluid. (d) Storage moduli $\left(G^{\prime}\right)$ and loss moduli $\left(G^{\prime \prime}\right)$ as a function of shear stress. (e) Optical image of a presentative rose-shaped pattern printed on a TPU substrate. (f) Optical and optical microscope images of an array of line-shaped features. (g) Optical images of pigeon-shaped patterns on various elastomer substrates including SIS (left), silicone (middle), and PVDF-HFP (right).

to TPU, the optimized yield-stress fluid is compatible with a variety of stretchable substrates, including SIS, silicone, and PVDF-HFP, as shown in Figure 1(g).

As revealed by the inset of Figure 2(a), the printed yieldstress fluid electrode is highly transparent primarily due to the high water content (>85 w/w \%). Figure 2 (a) shows optical transmittance as a function of sheet resistance controlled by adjusting the thickness of the electrode. The yield-stress fluid electrodes demonstrate competitive optoelectronic properties in terms of Rs $=400 \Omega /$ sq. at $T=98.4 \%$, Rs $=$ $220 \Omega$ /sq. at $T=94.2 \%$, and Rs $=90 \Omega /$ sq. at $T=83.5 \%$, respectively. In Figure $2(\mathrm{~b})$, the fairly flat transmittance spectra suggest color-neutral characteristics, which essentially eliminate chromatic corrections in device applications [55]. The attractive performances of these electrodes are largely associated with the exceptional conductivity of $14.4 \mathrm{~S} / \mathrm{m}$. In Figure 2(c), $10 \mathrm{M} \mathrm{LiCl}$ aqueous solution has abundant dissociated ions and a high conductivity of $16.2 \mathrm{~S} / \mathrm{m}$, which represents the upper limit for its derivatives. The addition of $5 \mathrm{w} / \mathrm{v} \%$ PEO gives rise to $\sim 10 \%$ decrease in conductivity as compared with that of the pristine aqueous electrolyte. The conductive liquid of the polymer solution is a Newtonian fluid with increased viscosity to hinder the ionic diffusions $[56,57]$. The subsequent incorporation of $10 \mathrm{w} / \mathrm{v} \%$ fumed silica gives rise to negligible changes in con- ductivity, because the colloid network is phase-separated from the electrolyte with limited influences on the ion transports [58]. In practice, the concentration of fumed silica is systematically modulated to achieve the optimal printing quality. The PEO is kept in the low concentration range due to the negative impact on the ion conductivity (Figure S6). Accordingly, the optimized yield-stress fluid functions as a high-performance ionic conductor by inheriting the excellent conductivity of the original aqueous electrolyte.

The water retention capability is a key requirement for stable long-term operations. The water loss is evaluated by monitoring the weight as a function of storage time in a dry environment. The rheological modifiers are ineffective to prevent the yield-stress fluid from drying out, as illustrated in Figure S7. The salt choices are critical for yieldstress fluids to stay hydrated. As shown in Figure 2(d), the water loss of LiCl-based fluid gradually approaches the steady-state value of $\sim 20 \%$. The exceptional water retention is further confirmed by the preservation of the overall morphology of the printed electrode after $50 \mathrm{~h}$ storage under dry air conditions (see the inset of Figure 2(d)). In contrast, continuous weight loss through evaporation is observed in NaCl-based fluid. The large differences in water retention are associated with the 

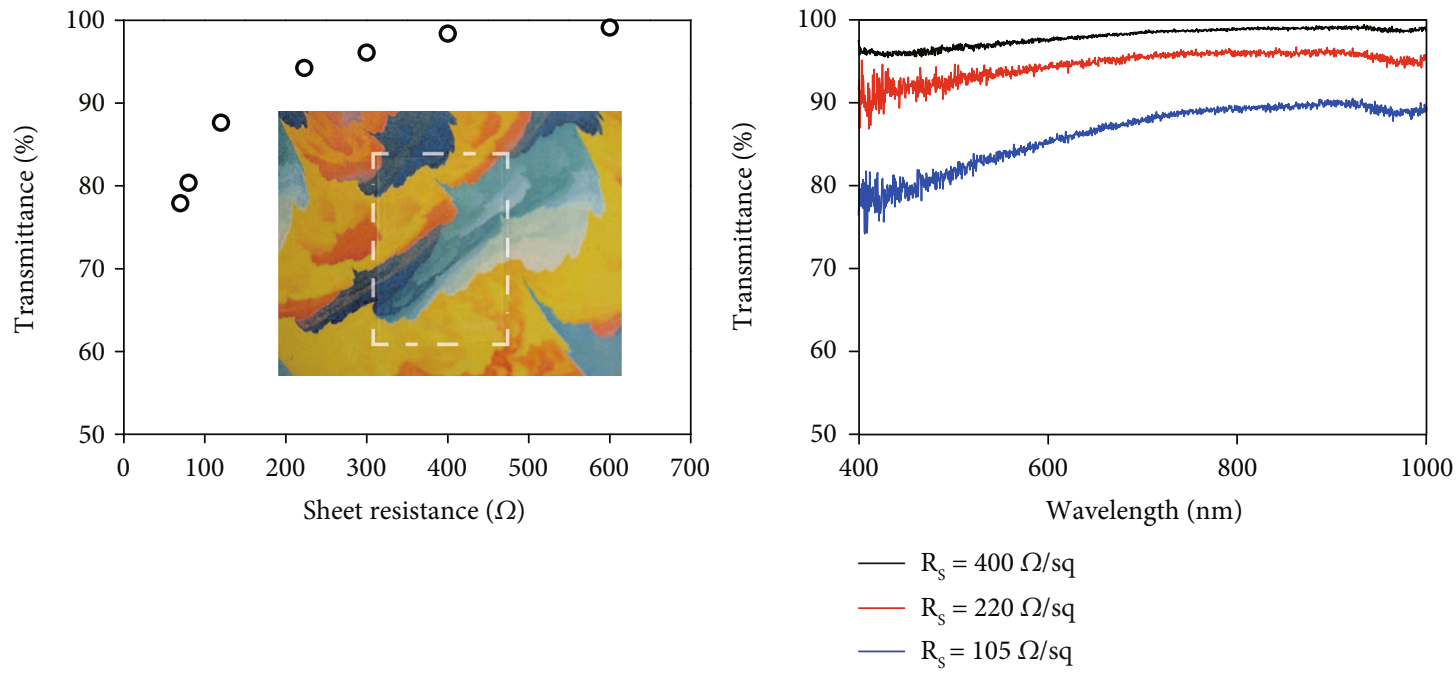

(a)

(b)

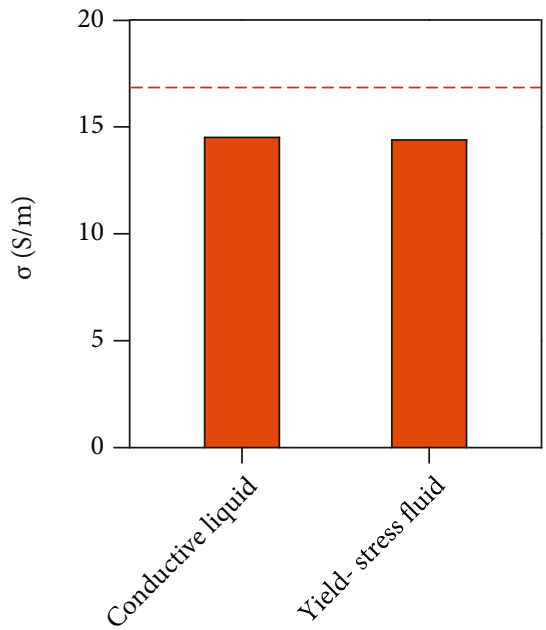

(c)



(d)

FIgURE 2: Yield-stress fluid electrodes as transparent conductors. (a) Transmittance spectra of electrodes with different sheet resistance in the wavelength range from 400 to $1000 \mathrm{~nm}$. Inset: Image of a representative transparent electrode with a sheet resistance of $400 \Omega$. (b) Optical transmittance (at $550 \mathrm{~nm}$ ) versus sheet resistance for electrodes based on the yield-stress fluid. (c) Conductivity of the conductive liquid and the yield-stress fluid. The conductivity of the original $10 \mathrm{M} \mathrm{LiCl}$ aqueous solution is marked by the dotted line for comparison. (d) Water loss as a function of storage time in an environmental chamber at $23^{\circ} \mathrm{C}$ and $20 \%$ relative humidity for yield-stress fluids based on $\mathrm{LiCl}$ and $\mathrm{NaCl}$. Inset: Image of a LiCl-based electrode acquired at 0 and $50 \mathrm{~h}$. Scale bar: $1 \mathrm{~cm}$.

variations in ionic hydration degrees of the dissolved salts [59]. The highly hydratable $\mathrm{LiCl}$ salt is well suited to improve water retention, because the strong bonds between cation/anion-water pairs provide energy barriers that prevent water molecules from evaporation [60]. In addition, the water retention capability is also directly correlated with $\mathrm{LiCl}$ concentration, as illustrated in Figure S7. The reduced water loss at high salt concentration is ascribed to the decreased fraction of free water molecules that readily evaporate.

The printed yield-stress fluid pattern stays as solid-state structures to enable facile encapsulation by elastomers, which functions as a highly deformable conductor confined in the elastomer. The as-prepared electrode is ultrastretch- able to reach large uniaxial tensile strain up to $700 \%$, as illustrated by the optical images in Figure 3(a). The exceptional deformability of the electrode is ascribed to the rapid transition into liquid-like behavior of yield-stress fluid upon tensile deformations, thereby resembling the functional liquids with the ultimate stretchability only limited by the fracture strain of the encapsulating elastomer [27]. The corresponding resistance shows a gradual increase in response to tensile deformations (Figure 3(b)). The normalized resistance follows the characteristic behavior of ideal conductive liquids expressed as $(1+\varepsilon)^{2}$, where $\varepsilon$ is the uniaxial strain (see Figure S8). As the reversible solid/liquid transitions completely preserve the high conductivity of the yieldstress fluid, the resistance change is purely a geometric 


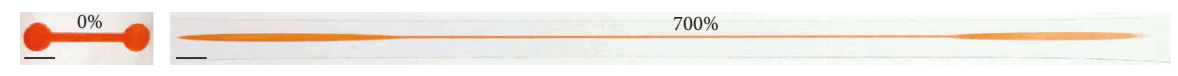

(a)



(b)



(c)

Figure 3: Yield-stress fluid electrode under uniaxial tensile deformations. (a) Optical images of a yield-stress fluid electrode at $0 \%$ and $700 \%$ strains. Scale bar: $5 \mathrm{~mm}$. (b) Normalized resistance as a function of strain. (c) Evolution of normalized resistance over 500 stretch relaxation cycles in the strain range from $100 \%$ to $400 \%$.

effect with the combined longitude extensions and crosssectional contractions (see Figure 8 and S9). The yieldstress fluid electrode is further evaluated by straincontrolled fatigue tests. Figure 3(c) shows the evolution of the normalized resistance over 500 cycles of tensile deformations in the range from $100 \%$ to $400 \%$ strains. Notice that the samples are only allowed to relax at $100 \%$ strain due to a fairly long time is required for the full recovery of the viscoelastic encapsulation to the original state after large tensile deformations [15]. The resistance shows extremely stable responses in spite of repetitive mechanical strains, which demonstrates exceptional durability attractive for practical implementations. The inherent immunity to mechanical fatigue essentially resembles that of liquid state conductors [61].

The yield-stress fluid electrodes are also compatible with large biaxial tensile deformations. In the inset of Figure 4, a series of optical images reveal the stable and uniform expansions of the electrodes. In Figure 4, the corresponding normalized resistance gradually increases with the area strain, which follows the predicted behavior of ideal liquids (see Figure S10 and S11). The transition into a liquid-state conductor results in well-expected resistance responses according to geometric deformations. The exceptional deformability of the yield-stress fluid electrode is essentially associated with the preservation of high ionic conductivity under various mechanical manipulations.

Highly stretchable yield-stress fluid electrode allows facile creation of transparent strain sensors for epidermal wearable electronics. The as-prepared transparent strain sensor is shown in Figure S12. In the inset of Figure 5, the electrode is colored in red for clear visualization of the patterning design consisting of interconnected parallel line segments. In Figure 5(a), the strain sensor was subjected to cyclic uniaxial tensile deformations with the peak strains progressively increased from $100 \%$ to $500 \%$. The resistance is directly correlated with the applied strain and negligibly affected by the strain history. The normalized resistance

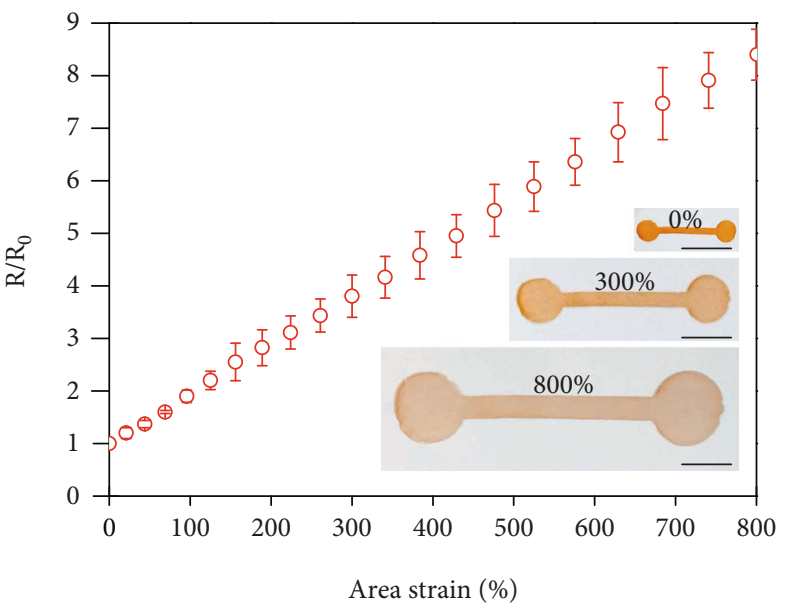

Figure 4: Yield-stress fluid electrode under biaxial tensile deformations. Normalized resistance as a function of area strain. Inset: optical images showing a yield-stress fluid electrode at $0 \%$, $300 \%$, and $800 \%$ area strains. Scale bar: $10 \mathrm{~mm}$.

and gauge factor are shown in Figure S13. In addition, the strain sensor exhibits fast and reliable responses to a series of step deformations, as shown in Figure 5(b). The durability is further demonstrated by the stable response curve after 1000 cycles of tensile deformations from 0 to $200 \%$ strains (see Figure S14). The strain sensor is readily attached to the skin by using silicone gel adhesive for monitoring the joint motions in real time, as shown in Figures $5(\mathrm{c})$ and $5(\mathrm{~d})$. The maximum strains are estimated as $30 \%$ for finger bending and $16 \%$ for wrist flexion, respectively. The strain sensor demonstrates fast and reproducible responses to large tensile strains, which represents a promising wearable sensor to detect complex body movements.

Compliant transparent electrodes also serve as key building components for emerging stretchable optoelectronics. As 


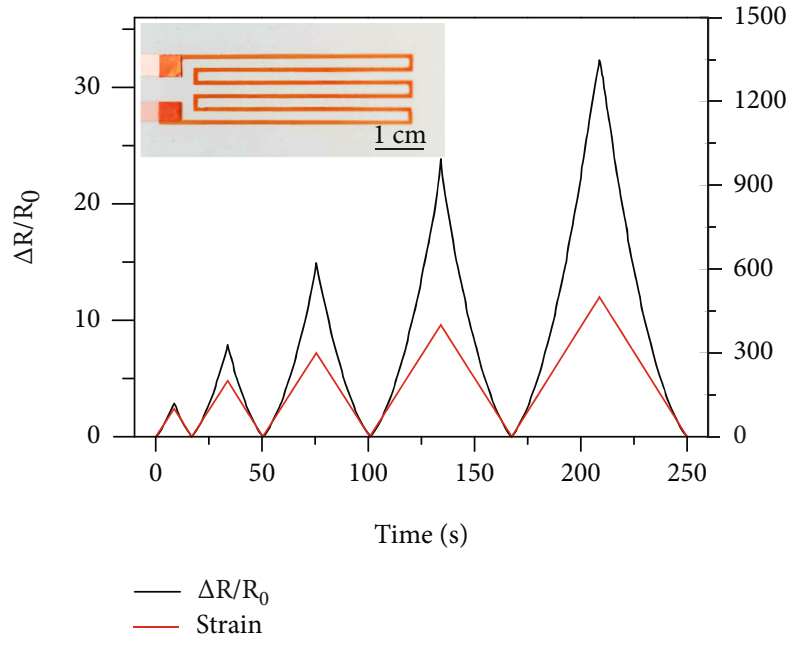

(a)

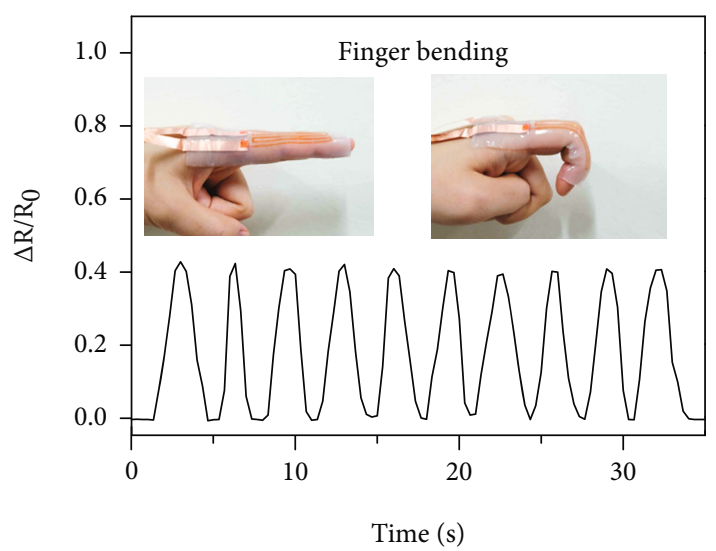

(c)

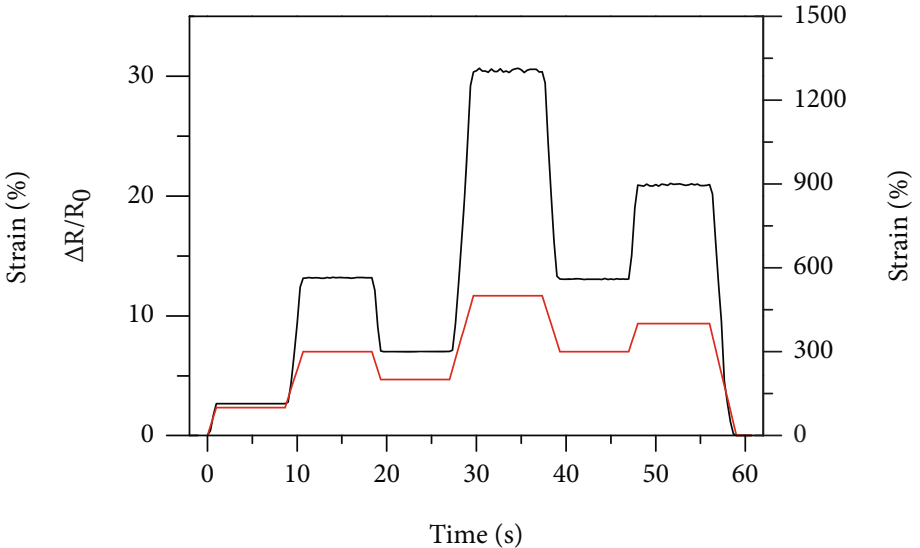

(b)

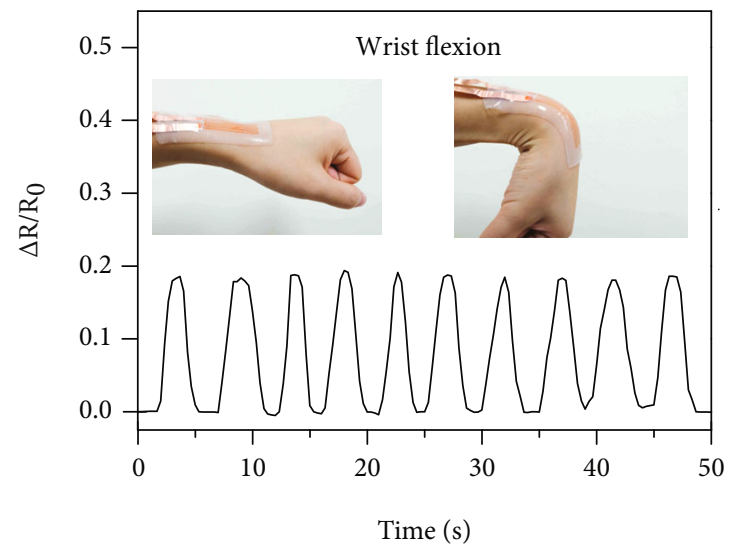

(d)

FIGURE 5: Strain sensors based on yield-stress fluid electrodes. (a) Normalized resistance change under cyclic uniaxial tensile deformations with the peak strains of $100 \%, 200 \%, 300 \%, 400 \%$, and 500\%. Inset: image of an as-prepared strain sensor. (b) Normalized resistance change in response to step deformations. (c) Real-time normalized resistance of the strain sensor to monitor dynamic motions of the index finger (c) and the wrist (d).

schematically illustrated in Figure 6(a), the yield-stress fluid electrodes are employed to prepare stretchable alternating current electroluminescent (ACEL) devices, in which the phosphor microparticles embedded in the electroluminescent layer are stimulated by alternative electric fields for light emissions. The luminous pattern is therefore defined by the overlapping area between the top and bottom transparent electrodes. The voltage-dependent emission characteristics are shown in Figure 6(c) for a representative ACEL device powered by a $25 \mathrm{kHz}$ square-wave voltage. The luminance is $3.83 \mathrm{~cd} / \mathrm{m}^{2}$ at $100 \mathrm{~V}, 35.76 \mathrm{~cd} / \mathrm{m}^{2}$ at $200 \mathrm{~V}$, and $111.2 \mathrm{~cd} /$ $\mathrm{m}^{2}$ at $320 \mathrm{~V}$, respectively. Despite a thick electroluminescent layer design, the device can provide luminance of $100 \sim 200 \mathrm{~cd} / \mathrm{m}^{2}$ at relatively low voltages typically required for indoor applications $[62,63]$, by using thermoplastic polyurethane as a polar dielectric matrix of the electroluminescent layer (see Figure S15). In addition, the luminance frequency characteristic with an applied voltage of amplitude $200 \mathrm{~V}$ is shown in Figure S16. The emission intensity initially increases with the frequency until $30 \mathrm{kHz}$ as a result of increased injection of accelerated electrons to activate the luminance centers [64-66]. The luminance decreases by further raising the frequency due to the capture of injected electrons at donors [67-69].

The deformability of the ACEL devices is evaluated by measuring their optoelectronic characteristics in response to various mechanical manipulations. In Figures 6(b), a series of optical images reveal a spiral-patterned device under uniaxial tensile deformations, which maintains stable and uniform light emission up to $700 \%$ strain. The dynamic deformation process of the ACEL device is further revealed in Movie S2. In Figure 6(d), the corresponding normalized luminance initially increases with tensile deformations until $300 \%$ strain, which is ascribed to enhanced electric fields by reducing the electroluminescent layer thickness. As the strain further increases, the light intensity continuously decreases due to the significant rise in resistance of the electrodes. Stretchable ACEL device is also assessed by repetitive uniaxial stretching in the strain range from $100 \%$ to $400 \%$, as shown in Figure 6(e). The intensity is maintained at $78 \%$ of 


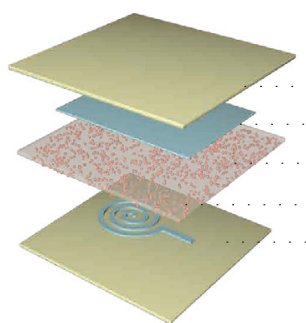

(a)

(c)



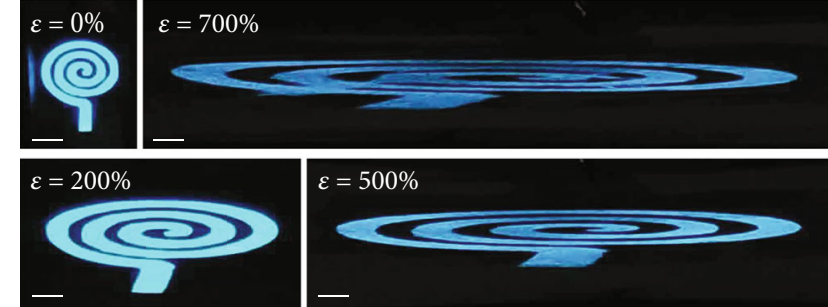

(b)

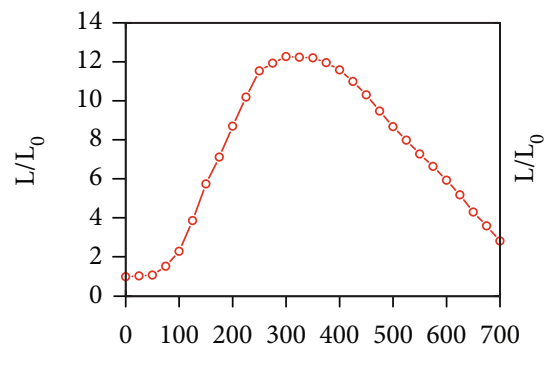

Strain (\%)

(d)

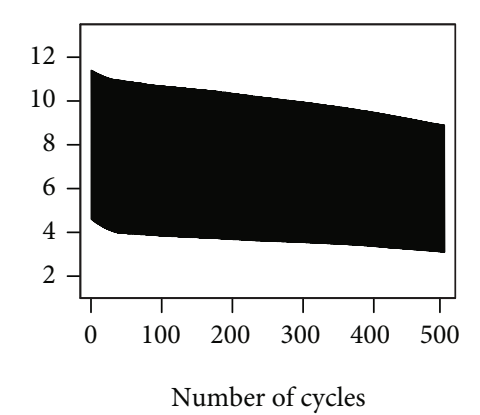

(e)

FiguRE 6: Stretchable alternating current electroluminescent (ACEL) device under uniaxial tensile deformations. (a) Schematic illustration of the ACEL device. (b) A series of optical images showing a representative device with a spiral-shaped luminous pattern at different uniaxial tensile strains. Scale bar: $5 \mathrm{~mm}$. (c) Luminance versus voltage amplitude of a stretchable ACEL device powered by square-wave voltages at $25 \mathrm{kHz}$. (d) Normalized luminance as a function of tensile strain. (e) Evolution of normalized emission intensity over 500 stretch relaxation cycles in the strain range from $100 \%$ to $400 \%$.

the initial value after 500 cycles, which verifies the excellent durability desirable for practical application settings. In addition to uniaxial stretching, the ACEL devices are capable of accommodating large biaxial tensile deformations. As illustrated by optical images in Figure 7, the device retains a stable and uniform dragonfly-shaped pattern up to $800 \%$ area strain. As the area expands, the corresponding emission intensity first increases and then decreases, with a peak value achieved at $340 \%$ area strain (see Figure 7 ). The trend also comes from the competition between the enhanced electric field and the increased electrode resistance.

\section{Discussions}

In summary, we have developed a yield-stress fluid by dispersing silica nanoparticles into a concentrated aqueous electrolyte, which functions as viscous ink for screen printing of highly conductive and ultrastretchable transparent conductors over various elastomer substrates. The printed features are solid state in nature with exceptional structural retention as a result of the three-dimensional colloid network. The transition into liquid-like behavior upon tensile deformation results in ultrahigh stretchability up to the fracture strain of the elastomer substrates. The practical suitability is demonstrated by wearable strain sensors and stretchable light-emitting devices. The yield-stress fluids allow facile and scalable fabrication of high-performing transparent conductors, which may find a broad range of applications in stretchable optoelectronic devices and systems.



FIgURE 7: Stretchable ACEL device under biaxial tensile deformations. Normalized emission intensity as a function of area strain. Inset: optical images showing a dragonfly-shaped luminous pattern at $0 \%, 300 \%$, and $800 \%$ area strains. Scale bar: $2 \mathrm{~cm}$.

\section{Materials and Methods}

4.1. Materials and Preparations. All chemical reagents were commercially acquired including polyethylene oxide (PEO, $M_{w}=300 \mathrm{k}$ ) from Shanghai Aladdin Bio-Chem Technology Co., Ltd., lithium chloride ( $\mathrm{LiCl})$ and sodium chloride $(\mathrm{NaCl})$ from Shanghai Energy Chemical Co., Ltd., and fumed silica (AEROSIL 380) from Evonik Degussa GmbH. Thermoplastic elastomers were purchased from corresponding vendors including thermoplastic polyurethane (TPU, 
Estane T460A) from Lubrizol Inc., styrene-isoprene-styrene (SIS, D1113) from Kraton Corporation, and PVDF-HFP (Daiel G801) from Daikin Industries. Silicone (Ecoflex 00$30 \mathrm{~A} / \mathrm{B})$ and polyurethane (PU, Clear Flex $30 \mathrm{~A} / \mathrm{B})$ were obtained from Smooth-On, Inc. Cu-doped $\mathrm{ZnS}$ phosphor microparticles were synthesized by Shanghai Keyan Phosphor Technology Co., Ltd. The VHB 4910 tape of $1 \mathrm{~mm}$ in thickness was obtained from $3 \mathrm{M}$ Inc. The ionically conductive liquid was prepared by dissolving high concentrations of selected salts in an aqueous PEO solution ( $5 \mathrm{w} / \mathrm{v} \%$ ), in terms of $4 \mathrm{~mol} / \mathrm{L}$ for $\mathrm{NaCl}$ and $10 \mathrm{~mol} / \mathrm{L}$ for $\mathrm{LiCl}$, which approached the solubility under the ambient conditions [60]. The yield-stress fluids were formed by further addition of fumed silica $(10 \mathrm{w} / \mathrm{v} \%)$ as a rheological modifier, followed by homogenization in a planetary ball mill (YXQM, Changsha MITR Co., Ltd.) for $2 \mathrm{~h}$. As regards the preparation of stretchable substrates, all thermoplastic elastomers were dissolved in selected solvents and then drop cast onto nonsticky glass wafers functionalized with octadecyltrichlorosilane (OTS), followed by natural evaporation to thoroughly remove the solvents. The solvent choices and concentrations were adjusted to achieve optimal quality of the resulting substrates, including toluene for tetrahydrofuran for TPU $(20 \mathrm{w} /$ $\mathrm{v} \%)$, SIS (20 w/v \%), and 4-Methyl-2-pentanone for PVDFHFP $(25 \mathrm{w} / \mathrm{v} \%)$. Silicone substrates were obtained by dropcasting mixed precursor of Ecoflex 00-30 A/B onto OTSmodified glass wafers and then thermally curing at $120^{\circ} \mathrm{C}$ for $2 \mathrm{~h}$. All printing processes were carried out on a manual screen printer (Zhuhai Kaivo Electronic Co., Ltd.) by using stainless steel stencil masks.

4.2. Material Characterizations. Structural characterizations were carried out by optical microscopy and confocal laser scanning microscopy by using a Keyence VK-X1000 microscope. Optical images were captured using a Fujifilm X-T10 digital camera. Rheology experiments were conducted on a HAAKE RheoStress 600 instrument. Steady and dynamic oscillatory shear measurements were conducted at room temperature by using a set of $35 \mathrm{~mm}$ diameter parallel plates with a sample thickness of $0.5 \mathrm{~mm}$. The frequency was fixed at $1 \mathrm{~Hz}$ in all the dynamic oscillatory shear measurements. The optical transmittance spectra were measured by a fiber optical spectrometer (Ideaoptics PG2000-Pro) equipped with a $6 \mathrm{~cm}$ diameter integrating sphere. The impedance of ionic conductors was measured using a GW Instek LCR Meter (Model LCR-6300). The storage stability was evaluated in an environmental chamber at $23^{\circ} \mathrm{C}$ and $20 \%$ relative humidity. The tensile deformations were applied by homemade motorized translation stages. The dielectric constants of elastomers were measured with parallel plate capacitor structures by the LCR Meter. The frequency-dependent dielectric constant of TPU is shown in Figure S15.

4.3. Strain Sensor Fabrication and Evaluation. A $100 \mu \mathrm{m}-$ thick silicone substrate was prepared on an OTS-modified glass wafer. The sensor electrode of $\sim 150 \mu \mathrm{m}$ in thickness was defined by stencil printing, followed by drop-casting a layer of Ecoflex 00-30 for encapsulation. The as-prepared strain sensor was brush painted with a layer of silicone gel
(Silbione RT GEL 4645A/B, Elkem Silicones) as the biocompatible adhesive for skin attachment. The resistance of the strain sensor was measured by the LCR meter. The uniaxial tensile strains were applied by a homemade motorized translation stage. The step deformations were applied at the maximal translation speed of the stage $(40 \mathrm{~mm} / \mathrm{s})$.

4.4. Light-Emitting Device Fabrication and Evaluation. A $100 \mu \mathrm{m}$-thick electroluminescent layer was prepared by doctor-blade coating with dissolved electroluminescent composite over OTS-modified glass. Spin cast TPU dielectric layers of $15 \mu \mathrm{m}$ in thickness were thermally laminated onto both sides of the electroluminescent layer to improve the dielectric strength. Subsequently, $\sim 150 \mu \mathrm{m}$-thick conductive electrode was generated by stencil printing and encapsulated by drop-cast PU precursor (Clear Flex 30 A/B). After curing under the ambient condition for $48 \mathrm{~h}$, the $\mathrm{VHB}$ tape was laminated on top as the substrate. The entire structure was peeled off from the glass wafer, flipped over, and attached to an OTS-modified glass wafer. The aforementioned printing and encapsulation steps were repeated to prepare the other transparent electrode. A high-voltage amplifier (Trek, Model 10/10B-HS) was employed to power the ACEL devices with well-defined waveforms supplied by a GW Instek AFG-2500 function generator. Emissive properties were quantified using a luminance and color meter (Konica Minolta CS-150).

\section{Data Availability}

All data needed to evaluate the conclusions in the paper are present in the paper and/or the Supplementary Materials. Additional data related to this paper may be requested from the authors.

\section{Conflicts of Interest}

There are no conflicts to declare.

\section{Authors' Contributions}

D.K. and X.W. conceived the experiments. Q.L. carried out material synthesis, structural characterizations, and physical property measurements. X.Y. performed rheological measurements. Q.L., Y.Z., and S.C. fabricated and evaluated the devices. All authors contributed to the scientific planning and discussions.

\section{Acknowledgments}

This work was supported by Key Research and Development Program of Jiangsu Provincial Department of Science and Technology of China (Grant No. BE2019002), National Natural Science Foundation of China (Grant No. 21790345), and High-Level Entrepreneurial and Innovative Talents Program of Jiangsu Province. 


\section{Supplementary Materials}

Supplementary 1. Figure S1: long-term stability of yieldstress fluids. Figure S2: influence of fumed silica concentration on the rheological properties. Figure S3: influence of PEO and silica additives on the rheological properties. Figure S4: influence of fumed silica concentration on the printing quality. Figure S5: influence of PEO concentration on the printing quality. Figure S6: conductivity of yieldstress fluid as a function of PEO concentration. Figure S7: influence of $\mathrm{LiCl}$ concentration on water retention. Figure S8: theoretical and experimental electromechanical properties of yield-stress fluid electrode under uniaxial tensile deformations. Figure S9: electromechanical properties of yield-stress fluid electrodes of different thicknesses under uniaxial tensile deformations. Figure 10: theoretical and experimental electromechanical properties of yield-stress fluid electrode under biaxial tensile deformations. Figure S11: electromechanical properties of yield-stress fluid electrodes of different thicknesses under biaxial tensile deformations. Figure S12: optical images of an as-printed transparent strain sensor. Figure S13: gauge factor of the strain sensor. Figure S14: durability of the strain sensor. Figure S15: dielectric constant of TPU elastomer. Figure S16: frequency-dependent luminance of a representative ACEL device.

Supplementary 2. Movie S1. A yield-stress fluid under static and shaking conditions.

Supplementary 3. Movie S2. A stretchable ACEL device retaining a stable luminous pattern under uniaxial deformation in the strain range from 0 to $700 \%$.

\section{References}

[1] J. A. Rogers, T. Someya, and Y. Huang, "Materials and mechanics for stretchable electronics," Science, vol. 327, no. 5973, pp. 1603-1607, 2010.

[2] Z. Suo, "Mechanics of stretchable electronics and soft machines," MRS Bulletin, vol. 37, no. 3, pp. 218-225, 2012.

[3] S. Wang, J. Y. Oh, J. Xu, H. Tran, and Z. Bao, "Skin-inspired electronics: an emerging paradigm," Accounts of Chemical Research, vol. 51, no. 5, pp. 1033-1045, 2018.

[4] Y. Chen, S. Lu, S. Zhang et al., "Skin-like biosensor system via electrochemical channels for noninvasive blood glucose monitoring," Science Advances, vol. 3, no. 12, article e1701629, 2017.

[5] D. Son, J. Lee, S. Qiao et al., "Multifunctional wearable devices for diagnosis and therapy of movement disorders," Nature Nanotechnology, vol. 9, no. 5, pp. 397-404, 2014.

[6] X. Wang, Y. Gu, Z. Xiong, Z. Cui, and T. Zhang, "Silk-molded flexible, ultrasensitive, and highly stable electronic skin for monitoring human physiological signals," Advanced Materials, vol. 26, no. 9, pp. 1336-1342, 2014.

[7] J. Kim, M. Lee, H. J. Shim et al., "Stretchable silicon nanoribbon electronics for skin prosthesis," Nature Communications, vol. 5, no. 1, 2014.

[8] B. C.-K. Tee, A. Chortos, A. Berndt et al., "A skin-inspired organic digital mechanoreceptor," Science, vol. 350, no. 6258, pp. 313-316, 2015.
[9] A. Chortos, J. Liu, and Z. Bao, "Pursuing prosthetic electronic skin,” Nature Materials, vol. 15, no. 9, pp. 937-950, 2016.

[10] J. W. Jeong, W. H. Yeo, A. Akhtar et al., "Materials and optimized designs for human-machine interfaces via epidermal electronics," Advanced Materials, vol. 25, no. 47, pp. 68396846, 2013.

[11] M. Wang, Z. Yan, T. Wang et al., "Gesture recognition using a bioinspired learning architecture that integrates visual data with somatosensory data from stretchable sensors," Nature Electronics, vol. 3, no. 9, pp. 563-570, 2020.

[12] J. Liang, L. Li, X. Niu, Z. Yu, and Q. Pei, "Elastomeric polymer light-emitting devices and displays," Nature Photonics, vol. 7, no. 10, pp. 817-824, 2013.

[13] C. Larson, B. Peele, S. Li et al., "Highly stretchable electroluminescent skin for optical signaling and tactile sensing," Science, vol. 351, no. 6277, pp. 1071-1074, 2016.

[14] X. Shi, Y. Zuo, P. Zhai et al., "Large-area display textiles integrated with functional systems," Nature, vol. 591, no. 7849, pp. 240-245, 2021.

[15] J. Wang, C. Yan, G. Cai, M. Cui, E. A. Lee-Sie, and L. P. See, "Extremely stretchable electroluminescent devices with ionic conductors," Advanced Materials, vol. 28, no. 22, pp. 44904496, 2016.

[16] Y. Zhou, C. Zhao, J. Wang et al., "Stretchable high-permittivity nanocomposites for epidermal alternating-current electroluminescent displays," ACS Materials Letters, vol. 1, no. 5, pp. 511-518, 2019.

[17] S. Jun, K. W. Choi, K.-S. Kim et al., "Stretchable photodetector utilizing the change in capacitance formed in a composite film containing semiconductor particles," Composites Science and Technology, vol. 182, p. 107773, 2019.

[18] F. Xu and Y. Zhu, "Highly conductive and stretchable silver nanowire conductors," Advanced Materials, vol. 24, no. 37, pp. 5117-5122, 2012.

[19] J. Liang, L. Li, K. Tong et al., "Silver nanowire percolation network soldered with graphene oxide at room temperature and its application for fully stretchable polymer lightemitting diodes," ACS Nano, vol. 8, no. 2, pp. 1590-1600, 2014.

[20] Y. Zhou, S. Cao, J. Wang et al., "Bright stretchable electroluminescent devices based on silver nanowire electrodes and high- $\mathrm{k}$ thermoplastic elastomers," ACS Applied Materials \& Interfaces, vol. 10, no. 51, pp. 44760-44767, 2018.

[21] C. Zhao, Y. Zhou, S. Gu et al., "Fully screen-printed, multicolor, and stretchable electroluminescent displays for epidermal electronics," ACS Applied Materials \& Interfaces, vol. 12, no. 42, pp. 47902-47910, 2020.

[22] B. S. Kim, J. B. Pyo, J. G. Son et al., "Biaxial Stretchability and transparency of ag nanowire 2D mass-spring networks prepared by floating compression," ACS Applied Materials \& Interfaces, vol. 9, no. 12, pp. 10865-10873, 2017.

[23] X. Ho, J. Nie Tey, W. Liu, C. Kweng Cheng, and J. Wei, "Biaxially stretchable silver nanowire transparent conductors," Journal of Applied Physics, vol. 113, no. 4, article 044311, 2013.

[24] J.-B. Chossat, Y.-L. Park, R. J. Wood, and V. Duchaine, “A soft strain sensor based on ionic and metal liquids," IEEE Sensors Journal, vol. 13, no. 9, pp. 3405-3414, 2013.

[25] Z. Ma, B. Su, S. Gong et al., "Liquid-wetting-solid strategy to fabricate stretchable sensors for human-motion detection," ACS Sensors, vol. 1, no. 3, pp. 303-311, 2016. 
[26] Y. Wang, S. Gong, S. J. Wang, G. P. Simon, and W. Cheng, "Volume-invariant ionic liquid microbands as highly durable wearable biomedical sensors," Materials Horizons, vol. 3, no. 3, pp. 208-213, 2016.

[27] H. Ota, K. Chen, Y. Lin et al., "Highly deformable liquid-state heterojunction sensors," Nature Communications, vol. 5, no. 1, p. 5032, 2014.

[28] J. W. Kim and J. M. Myoung, "Flexible and transparent electrochromic displays with simultaneously implementable subpixelated ion gel-based viologens by multiple patterning," Advanced Functional Materials, vol. 29, no. 13, article 1808911, 2019.

[29] S. Li, B. N. Peele, C. M. Larson, H. Zhao, and R. F. Shepherd, "A stretchable multicolor display and touch Interface using Photopatterning and transfer printing," Advanced Materials, vol. 28, no. 44, pp. 9770-9775, 2016.

[30] C. Keplinger, J. Y. Sun, C. C. Foo, P. Rothemund, G. M. Whitesides, and Z. Suo, "Stretchable, transparent, ionic conductors," Science, vol. 341, no. 6149, pp. 984-987, 2013.

[31] J. Y. Sun, C. Keplinger, G. M. Whitesides, and Z. Suo, "Ionic skin," Advanced Materials, vol. 26, no. 45, pp. 7608-7614, 2014.

[32] F. Zare Bidoky, B. Tang, R. Ma et al., "Sub-3 V ZnO electrolyte-gated transistors and circuits with screen-printed and photo-crosslinked ion gel gate dielectrics: new routes to improved performance," Advanced Functional Materials, vol. 30, no. 20, p. 1902028, 2019.

[33] J. Y. Sun, X. Zhao, W. R. Illeperuma et al., "Highly stretchable and tough hydrogels," Nature, vol. 489, no. 7414, pp. 133-136, 2012.

[34] T. L. Sun, T. Kurokawa, S. Kuroda et al., "Physical hydrogels composed of polyampholytes demonstrate high toughness and viscoelasticity," Nature Materials, vol. 12, no. 10, pp. 932-937, 2013.

[35] H. Yuk, T. Zhang, S. Lin, G. A. Parada, and X. Zhao, "Tough bonding of hydrogels to diverse non-porous surfaces," Nature Materials, vol. 15, no. 2, pp. 190-196, 2016.

[36] H. Yuk, T. Zhang, G. A. Parada, X. Liu, and X. Zhao, "Skininspired hydrogel-elastomer hybrids with robust interfaces and functional microstructures," Nature Communications, vol. 7, no. 1, p. 12028, 2016.

[37] D. Bonn, M. M. Denn, L. Berthier, T. Divoux, and S. Manneville, "Yield stress materials in soft condensed matter," Reviews of Modern Physics, vol. 89, no. 3, article 035005, 2017.

[38] M. Mariya and J. Nikolay, "Creating a yield stress in liquid oils by the addition of crystallisable modifiers," Journal of Food Engineering, vol. 51, no. 3, pp. 235-237, 2002.

[39] A. Tárrega, E. Costell, and M. A. Rao, "Vane yield stress of native and cross-linked starch dispersions in skimmed milk: effect of starch concentration and $\lambda$-carrageenan addition," Food Science and Technology International, vol. 12, no. 3, pp. 253-260, 2016.

[40] B. Y. Ahn, E. B. Duoss, M. J. Motala et al., "Omnidirectional printing of flexible, stretchable, and spanning silver microelectrodes," Science, vol. 323, no. 5921, pp. 1590-1593, 2009.

[41] B. M. Rauzan, A. Z. Nelson, S. E. Lehman, R. H. Ewoldt, and R. G. Nuzzo, "Particle-free emulsions for 3D printing elastomers," Advanced Functional Materials, vol. 28, no. 21, article 1707032, 2018.

[42] C. B. Highley, K. H. Song, A. C. Daly, and J. A. Burdick, "Jammed microgel inks for 3D printing applications," Advanced Science (Weinh), vol. 6, no. 1, p. 1801076, 2019.
[43] C. H. Lee, V. Moturi, and Y. Lee, "Thixotropic property in pharmaceutical formulations," Journal of Controlled Release, vol. 136, no. 2, pp. 88-98, 2009.

[44] J. E. Mealy, J. J. Chung, H. H. Jeong et al., "Injectable granular hydrogels with multifunctional properties for biomedical applications," Advanced Materials, vol. 30, no. 20, article e1705912, 2018.

[45] A. Z. Nelson and R. H. Ewoldt, "Design of yield-stress fluids: a rheology-to-structure inverse problem," Soft Matter, vol. 13, no. 41, pp. 7578-7594, 2017.

[46] A. Z. Nelson, K. S. Schweizer, B. M. Rauzan, R. G. Nuzzo, J. Vermant, and R. H. Ewoldt, "Designing and transforming yield-stress fluids," Current Opinion in Solid State and Materials Science, vol. 23, no. 5, article 100758, 2019.

[47] M. Kawaguchi, "Dispersion stabilities and rheological properties of fumed silica suspensions," Journal of Dispersion Science and Technology, vol. 38, no. 5, pp. 642-660, 2017.

[48] N. B. Uriev and I. Y. Ladyzhinsky, "Fractal models in rheology of colloidal gels," Colloids and Surfaces A: Physicochemical and Engineering Aspects, vol. 108, no. 1, pp. 1-11, 1996.

[49] M. Kawaguchi, T. Yamamoto, and T. Kato, "Rheological properties of silica suspensions in aqueous solutions of block copolymers and their water-soluble components," Journal of Colloid and Interface Science, vol. 241, no. 1, pp. 293-295, 2001.

[50] D. Heath and T. F. Tadros, "Influence of $\mathrm{pH}$, electrolyte, and poly(vinyl alcohol) addition on the rheological behavior of aqueous silica (Aerosil) dispersions," Journal of Colloid and Interface Science, vol. 93, no. 2, pp. 320-328, 1983.

[51] E. H. Amalu, N. N. Ekere, and S. Mallik, "Evaluation of rheological properties of lead-free solder pastes and their relationship with transfer efficiency during stencil printing process," Materials \& Design, vol. 32, no. 6, pp. 3189-3197, 2011.

[52] P. G. Karagiannidis, S. A. Hodge, L. Lombardi et al., "Microfluidization of graphite and formulation of graphene-based conductive inks," ACS Nano, vol. 11, no. 3, pp. 2742-2755, 2017.

[53] K. Arapov, E. Rubingh, R. Abbel, J. Laven, G. de With, and H. Friedrich, "Conductive screen printing inks by gelation of graphene dispersions," Advanced Functional Materials, vol. 26, no. 4, pp. 586-593, 2016.

[54] C. Zhang, L. McKeon, M. P. Kremer et al., "Additive-free MXene inks and direct printing of micro-supercapacitors," Nature Communications, vol. 10, no. 1, p. 1795, 2019.

[55] H. Wu, D. Kong, Z. Ruan et al., "A transparent electrode based on a metal nanotrough network," Nature Nanotechnology, vol. 8, no. 6, pp. 421-425, 2013.

[56] J. Komiyama and R. M. Fuoss, "Conductance in water-poly(vinyl alcohol) mixtures," Proceedings of the National Academy of Sciences of the United States of America, vol. 69, no. 4, pp. 829833, 1972.

[57] K. S. Stojilkovic, A. M. Berezhkovskii, V. Y. Zitserman, and S. M. Bezrukov, "Conductivity and microviscosity of electrolyte solutions containing polyethylene glycols," The Journal of Chemical Physics, vol. 119, no. 13, pp. 6973-6978, 2003.

[58] K. Ueno, K. Hata, T. Katakabe, M. Kondoh, and M. Watanabe, "Nanocomposite ion gels based on silica nanoparticles and an ionic liquid: ionic transport, viscoelastic properties, and microstructure," The Journal of Physical Chemistry. B, vol. 112, no. 30, pp. 9013-9019, 2008. 
[59] R. H. Stokes and R. A. Robinson, "Ionic hydration and activity in electrolyte solutions," Journal of the American Chemical Society, vol. 70, no. 5, pp. 1870-1878, 1948.

[60] Y. Bai, B. Chen, F. Xiang, J. Zhou, H. Wang, and Z. Suo, "Transparent hydrogel with enhanced water retention capacity by introducing highly hydratable salt," Applied Physics Letters, vol. 105, no. 15, article 151903, 2014.

[61] S. H. Zhang, F. X. Wang, J. J. Li, H. D. Peng, J. H. Yan, and G. B. Pan, "Wearable wide-range strain sensors based on ionic liquids and monitoring of human activities," Sensors (Basel), vol. 17, no. 11, p. 2621, 2017.

[62] S. Steudel, K. Myny, S. Schols et al., "Design and realization of a flexible QQVGA AMOLED display with organic TFTs," Organic Electronics, vol. 13, no. 9, pp. 1729-1735, 2012.

[63] M. S. White, M. Kaltenbrunner, E. D. Głowacki et al., "Ultrathin, highly flexible and stretchable PLEDs," Nature Photonics, vol. 7, no. 10, pp. 811-816, 2013.

[64] S. Tanaka, H. Kobayashi, H. Sasakura, and Y. Hamakawa, "Evidence for the direct impact excitation of Mn centers in electroluminescent ZnS:Mn films," Journal of Applied Physics, vol. 47, no. 12, pp. 5391-5393, 1976.

[65] W. A. Thornton, "Electroluminescence in zinc sulfide. Physical," Review, vol. 103, no. 5, pp. 1585-1586, 1956.

[66] H. Yang, P. H. Holloway, and B. B. Ratna, "Photoluminescent and electroluminescent properties of $\mathrm{Mn}$-doped $\mathrm{ZnS}$ nanocrystals," Journal of Applied Physics, vol. 93, no. 1, pp. 586-592, 2003.

[67] L. N. Tripathi, B. R. Chaubey, and C. P. Mishra, "Photo and electroluminescence properties in Zns: (cu, La) and Zns: (ag, La) phosphors," Pramana, vol. 16, no. 2, pp. 155-164, 1981.

[68] T. Taki and H. Bō, "The decay behavior of photo-induced electron spin resonance of chromium in zinc sulfide," Journal of the Physical Society of Japan, vol. 25, no. 5, pp. 1324-1329, 1968.

[69] J. Ibañez, E. Garcia, L. Gil, M. Mollar, and B. Marı', "Frequency-dependent light emission and extinction of electroluminescent $\mathrm{ZnS}: \mathrm{Cu}$ phosphor," Displays, vol. 28, no. 3, pp. 112-117, 2007. 